\title{
Non-linear adaptive image enhancement in wireless sensor networks based on non- subsampled shearlet transform
}

\author{
Ying Tong ${ }^{1,2^{*}}$ (D) and Jin Chen ${ }^{1,2^{*}}$
}

\begin{abstract}
Acquiring clear images is a crucial precondition in many image-related applications, such as wireless sensor network, industrial inspection, and machine vision. In this paper, a multi-scale image adaptive enhancement algorithm for image sensors in wireless sensor networks based on non-subsampled shearlet transform is presented. The images are decomposed into different scales of coefficients. Then the coefficients are enhanced by a non-linear enhancement function. We set two thresholds for this function. One is used to classify the coefficients between the set to be denoised and the set to be enhanced; the other is to control the enhanced intensity of the coefficients. The thresholds are selected adaptively according to the decomposition scale and the standard deviation of the coefficients. The performance of the proposed algorithm is evaluated both objectively and subjectively. And the results show that the visibility of the images is enhanced significantly.
\end{abstract}

Keywords: Image enhancement, Non-subsampled shearlet transform, Wireless sensor networks, Multi-scale analysis

\section{Introduction}

In wireless sensor networks for the situation understanding [1], different modalities are needed such as radar and optical images. In [2], it was shown that radar and images are two independent modalities which can be treated independently. In this paper, we focus on image modality in wireless sensor networks. The visibility and contrast of the captured images may be affected and may be degraded by many reasons, such as poor environmental condition, camera sensor noise, and other uncertain factors $[3,4]$. It is a necessary step before further processing and understanding the images to improve image quality with enhancement and denoising algorithm in many vision applications [5, 6]. The contrast and detail of images can be improved remarkably by those algorithms, so it will be easier for human or machine to identify and understand.

The image enhancement algorithms can be divided into two categories: one is spatial domain algorithm, which enhances the images by recalculating the gray

\footnotetext{
*Correspondence: tongying2334@163.com; cjwoods@163.com ${ }^{1}$ Tianjin Key Laboratory of Wireless Mobile Communications and Power Transmission, Tianjin Normal University, Tianjin 300387, China Full list of author information is available at the end of the article
}

value of the pixels. The other is transform domain algorithm, which enhances the images by adjusting the image coefficients in some transform domain.

At present, the multi-scale analysis theories (MAT) have been widely used in transform domain image enhancement algorithms. The main idea of MAT is to enhance the image details in different scales and directions. The key points of these algorithms are the selection of multiscale analysis tools and coefficients enhancement models.

In image processing applications, the most well-known multi-scale analysis tools are wavelet [7], contourlet [8], ridgelet [9], and other transform methods. Shearlet transform is a new multi-scale analysis tool which integrates the advantages of wavelet and contourlet [10]. It is well adapted to represent anisotropic features. Shearlet can provide an optimally sparse representation for multidimensional data [11], so it suits well for image processing tasks. The traditional multi-scale transforms tend to introduce pseudo-Gibbs artifacts owing to the down sampling operations. To eliminate this phenomenon, the shiftinvariant non-subsampled transforms were proposed. Non-subsampled shearlet transform (NSST) can capture the geometrical structure of an image, such as edges, much more precisely than those traditional multi-scale 
transforms. The decomposition procedure for NSST is similar to non-subsampled contourlet transform (NSCT). But the computational cost and complexity of NSST is much lower than that of NSCT $[12,13]$. And the number of directions for shearing has no limitation in the process of NSST, while the number of directions for NSCT decomposing must be any power of 2 . So NSST has more flexibility in the description of the geometric structure of the image.

We apply NSST to image enhancement algorithm in this paper. The shearlet coefficients are processed by a non-linear function which can suppress the noise coefficients and can strengthen the weak detail coefficients. Since the thresholds of the function are selected adaptively, the coefficients of each scale and direction are enhanced. The enhanced results of the proposed algorithm show a good performance on both the enhancement of the image details and the suppression of noise.

\section{Image enhancement based on NSST}

\subsection{Enhancement function and its properties}

The aim for enhancing images is to improve the quality of the images so that image perceptions required by further processes can be obtained. So a well-designed enhancement algorithm can amplify the details of image, can adjust the image contrast, and meanwhile, can remove the noise of signals. It means that the coefficients of the image should be processed separately. So it is a good choice to use the non-linear function as the enhancement model.

The enhancement model used in our enhancement algorithm is proposed by A.F. Laine in 1996 [14]. Since then, many scholars have applied this function to image enhancement processing and have achieved good results.

$$
f(x)=a[\operatorname{sigm}(c(x-b))-\operatorname{sigm}(-c(x+b))]
$$

Where $a=\frac{1}{\operatorname{sigm}(c(1-b))-\operatorname{sigm}(-c(1+b))}$, sigm is defined as $\operatorname{sigm}(x)=\frac{1}{1+e^{-x}}$ 。

There are two parameters named $c$ and $b$ in this function, where $b$ is used to control the enhancement range, and its value range is $(0,1)$; while $c$ is used to control the enhancement intensity, which usually takes the fixed value between 20 and 50. In the existing researches, many enhancement algorithms took the selection of the two parameters of $b$ and $c$ as key factors to improve the non-linear enhancement effect. Figure 1 demonstrates the curves with different $b$ and $c$ values in different colors. As shown in Fig. 1, the shape of the enhancement function varies greatly with different $b$ and $c$ values. At the same time, it also can be found that (take the positive direction of $\mathrm{X}$ axis as an example) two special points play important roles in the form of the function curve. One point is the first intersection between the curve and the line $f(x)=x$. It determines whether the image coefficient should be suppressed by denoising or enhanced by amplifying. It means that the horizontal coordinate of the point is the boundary of denoising and enhancement. The other important point is the intersection between the function curve and line $y=1$, it determines the extent of the enhancement. The coefficients with greater value than the horizontal coordinate of the point will be increased to the maximum value of the coefficients, while the smaller coefficients will be amplified non-linearly according to the function curve.

The two important points on the function curve, in this paper, are proposed as two thresholds to determine the form of the enhancement function. The

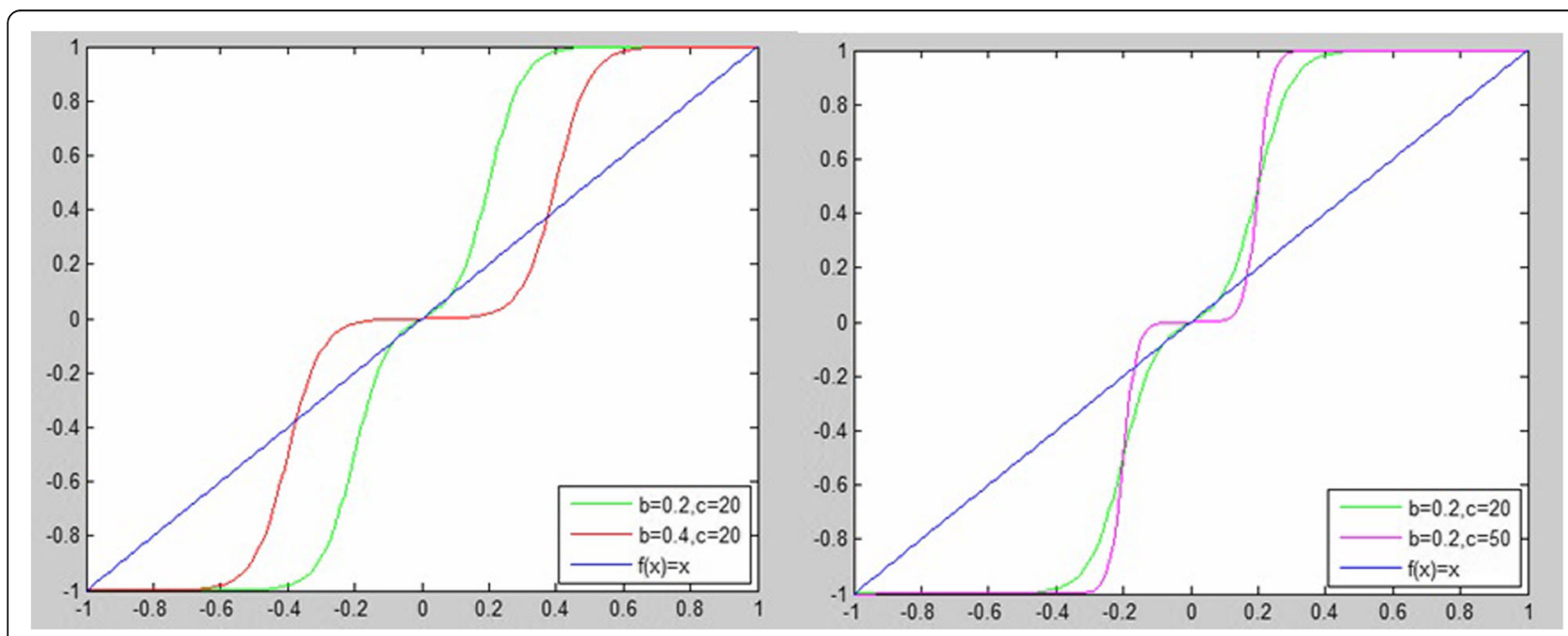

Fig. 1 The comparison of the function curves with different value of $b$ and $c$ 
thresholds will be selected adaptively according to the image multi-resolution decomposition coefficients of different scales and directions.

\subsection{Selection of the thresholds}

We set two thresholds of $T_{k}^{l}$ low and $T_{k}^{l}$ high in the enhancement function. $T_{k}^{l}$ low is the threshold to distinguish the coefficients between the enhancement set and the suppression set, $T_{k}^{l}$ high is used to control the contrast stretching degree of the enhanced coefficients. The two thresholds correspond to the two special points in the enhancement curves analyzed in 2.1.

Shearlet transform can decompose images into different scales and directions. And the coefficients of different scale have different features. The highfrequency coefficients often correspond to the image edges and details. This kind of coefficient needs obvious enhancement, but noise signals also mostly exist in the high-frequency coefficients. So it is required to suppress the high-frequency coefficients which have smaller absolute values. The low-frequency coefficients correspond to the image background or smooth parts, such coefficients should not be sharply enhanced but should try to maintain its contrast. Since there is less noise signals in low-frequency coefficients, their demand for denoising is not obvious. Therefore, we will set the thresholds for different decomposition scales and directions adaptively.

$T_{k}^{l}$ low corresponds to the horizontal coordinate of the enhancement function curve at the point of $x=f(x)$. It is supposed that $T_{k}^{l}$ low is proportional to the standard deviation of the decomposition coefficients, and the standard deviation calculation function satisfies formula (2).

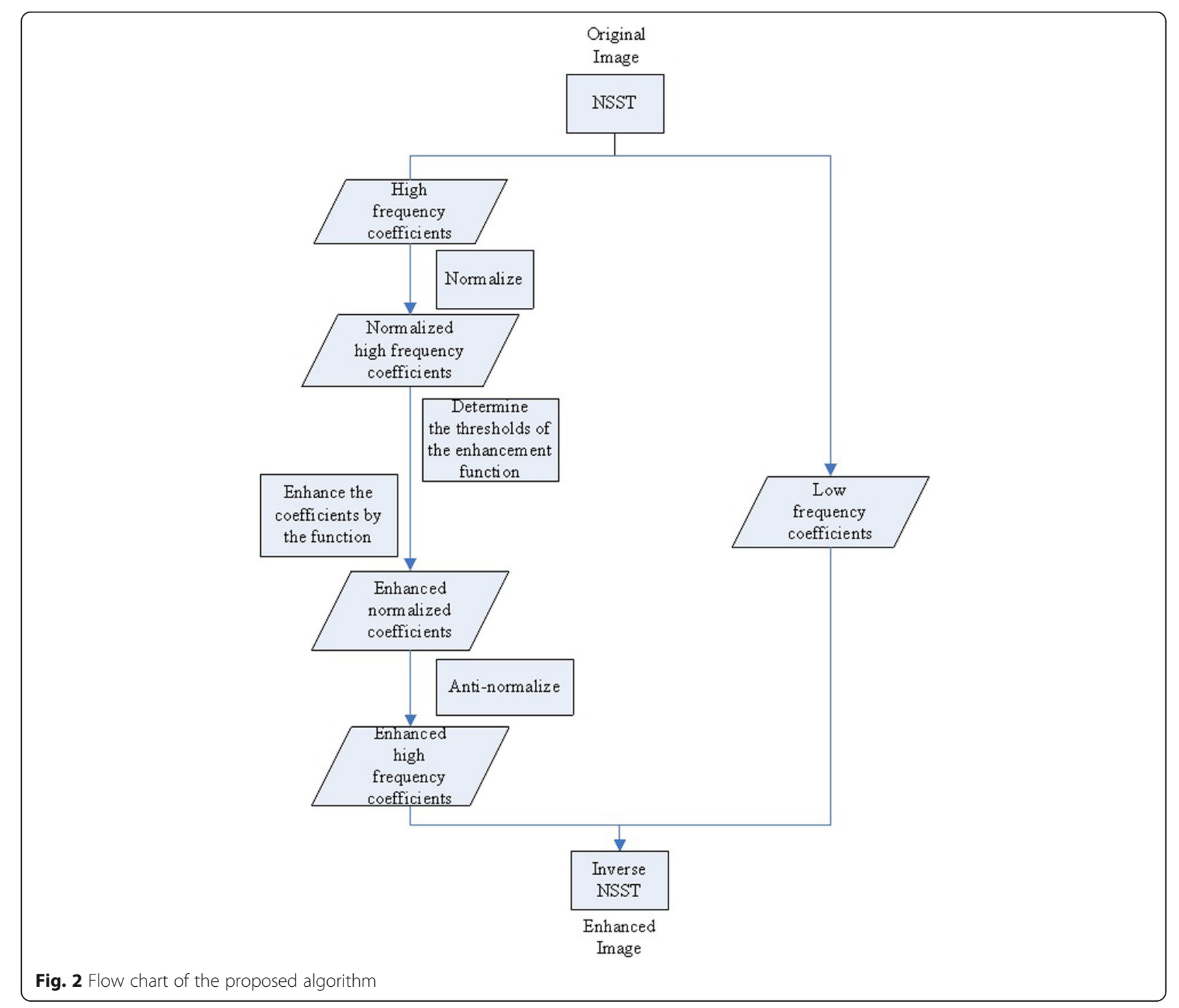




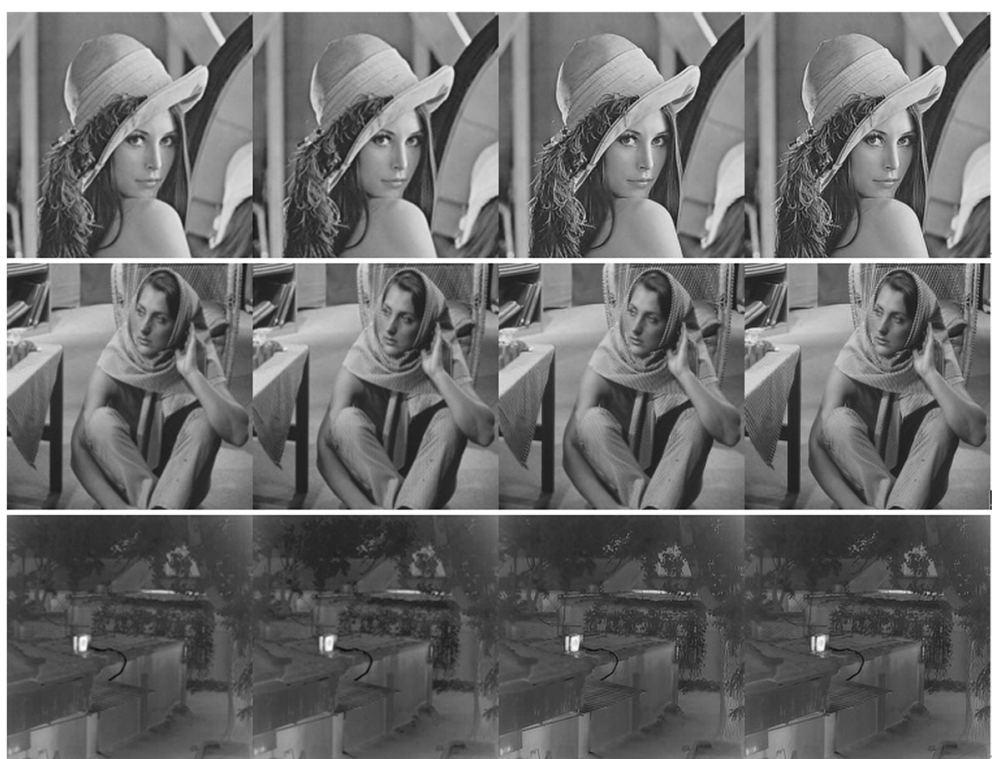

Fig. 3 The performance of different enhancement algorithms on grayscale images

$$
\sigma=\sqrt{\frac{1}{\mathrm{MN}} \sum_{m=1}^{M} \sum_{n=1}^{N}\left(c_{k}^{l}(m, n)-\mathrm{mean}_{c}\right)^{2}}
$$

Where $\sigma$ is the standard deviation of the coefficients; $c_{k}^{l}(m, n)$ is the value of the coefficient in the position of $(m, n)$; mean $n_{c}$ is the mean value of the coefficients of $k$ scale $l$ direction.

The frequency of decomposition coefficient rises with the increase of the decomposition scales. Noise signals are more likely to be gathered in the high-scale coefficients. So the calculation of $T_{k}^{l}$ low needs to consider the scale factors. The calculation function of $T_{k}^{l}$ low is shown as formula (3).

$$
T_{k \text { low }}^{l}=\frac{1}{8} \sigma * \text { level }
$$

Where $T_{k}^{l}$ low represents the first threshold of the coefficients in $k$ scale $l$ direction, $\sigma$ is the standard deviation of the decomposition coefficients, level is the decomposition scale. Formula (3) means that the value of $T_{k}^{l}$ low is propositional to the decomposition scale. That is, the greater the value of the decomposition scale, the more stringent the coefficients denoising.

$T_{k \text { high }}^{l}$ corresponds to the value of $\frac{9}{c}+b$ because from this point the derivative of the enhancement function begins to approach zero [15]. Its calculation function satisfies formula (4)

$$
T_{k \text { high }}^{l}=T_{\text {low }}^{l} * n / \text { level }^{2}
$$

Where $T_{k \text { high }}^{l}$ represents the second threshold of the coefficients in $k$ scale $l$ direction; $n$ is a constant, it is assigned to 150 in our experiments; $T_{k \text { high }}^{l}$ is inversely proportional to the decomposition scale. That is, the higher the frequency of the decomposition coefficient, the stronger the enhancement degree of the coefficient. While for the low-frequency coefficient, the enhancement curve tends to be gentle.

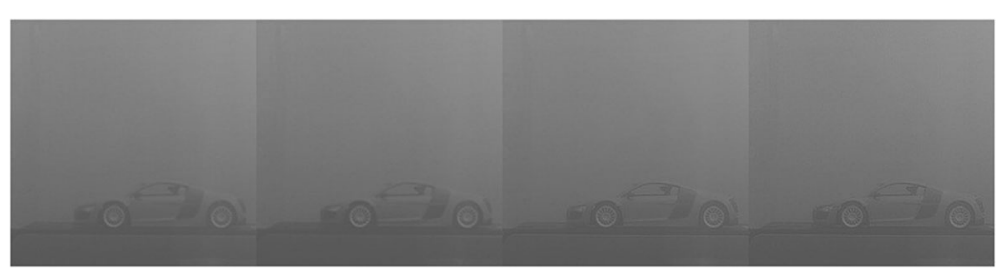

Fig. 4 The performance of different enhancement algorithms on noisy image 
Table 1 Objective evaluation parameters for Lena image set

\begin{tabular}{lllc}
\hline Lena & Cll & EPI & Running time(ms) \\
\hline Wavelet & 1.0295 & 1.0771 & 3.201676 \\
NSCT & 1.0639 & 2.0942 & 85.199992 \\
NSST & 1.0734 & 2.3440 & 5.709889 \\
\hline
\end{tabular}

\subsection{The overall framework of the algorithm}

A flow chart of the proposed enhancement algorithm is illustrated in Fig. 2, which is composed of seven steps:

(1)Decompose the original image by NSST to $K$ levels and $L$ orientations.

(2) Normalize the two highest frequency scales of coefficients in order to prepare them to be enhanced.

(3) Calculate the standard variance of the normalized coefficients in each layer and direction.

(4) Calculate the two enhancement thresholds for each layer and direction and use the thresholds to determine the form of the enhancement function.

(5) Enhance the normalized coefficients by the enhancement function.

(6) Anti-normalize the enhanced coefficients.

(7) Recompose the image by the enhanced highfrequency coefficients and the original lowfrequency coefficients.

Our algorithm is suitable to enhance grayscale images. Since it can suppress noise signals by the first threshold, it can also be used to enhance noisy images.

\section{Experiment results and discussion}

A set of grayscale images is used to demonstrate the performance of the proposed algorithm. The images include the standard 8-bit grayscale images of Lena, Barbara, and an infrared image. The hardware platform of the experiment is a desktop computer with 3.2 GHz CPU and 4 G RAM.

NSST, NSCT, wavelet are selected as image multiresolution decomposition tools. The enhancement effects and evaluation parameters are shown in Fig. 3 and Tables 1, 2, and 3. Among the four images of each set, the first one is the original image, the second one is
Table 3 Objective evaluation parameters for the infrared image set

\begin{tabular}{lllc}
\hline Infrared image & Cll & EPI & Running time $(\mathrm{ms})$ \\
\hline Wavelet & 1.1026 & 1.0318 & 3.903247 \\
NSCT & 1.2514 & 2.1303 & 86.213432 \\
NSST & 1.2542 & 2.5644 & 8.812085 \\
\hline
\end{tabular}

enhanced by wavelet soft threshold enhancement algorithm, the third one is enhanced by NSCT adaptive enhancement algorithm [16], and the last one is enhanced by the proposed algorithm.

By contrast, we can find that the performance of NSST and NSCT is much better than that of wavelet by human eyes, especially at the detail parts. We choose three evaluation parameters to evaluate the enhancement methods. They are contrast improvement index (CII) [17], edge preservation index (EPI) [18], and running time. The objective evaluation results listed in Tables 1, 2 , and 3 show that the proposed algorithm improves the image contrast and maintains the details. The running time of the program is acceptable.

An infrared image taken under haze environment is used to measure the ability of the algorithms to suppress noise while enhancing details. We choose equivalent number of looks (ENL) [19] as an extra evaluation parameters to testify the algorithms' ability to suppress noise. It can be seen from Fig. 4 and Table 4 that the proposed algorithm has the smallest ENL and biggest CII and EPI among the three. So it means that our algorithm has good enhancement effect on noisy images.

\section{Conclusions}

NSST can be used as a promising method in image processing tasks, such as denosing, fusion, enhancement. We propose a non-linear enhancement algorithm in wireless sensor networks. Two thresholds of the enhancement function are selected adaptively according to the standard variation of the coefficients and the decomposition scale. Experiment results show that our method performs well on the enhancement of both standard gray images and infrared image. And it can also be used to enhance noisy images since it has a good ability of suppress noises among signals.
Table 2 Objective evaluation parameters for Barbara image set

\begin{tabular}{lllc}
\hline Barbara & Cll & EPI & Running time $(\mathrm{ms})$ \\
\hline Wavelet & 1.0189 & 1.0172 & 3.190038 \\
NSCT & 1.1463 & 1.8084 & 86.739811 \\
NSST & 1.1961 & 2.0732 & 5.205702 \\
\hline
\end{tabular}

Table 4 Objective evaluation parameters for the noisy image set

\begin{tabular}{llll}
\hline Noisy image & Cll & EPI & ENL \\
\hline Wavelet & 1.0239 & 0.9223 & 6.9334 \\
NSCT & 1.0149 & 2.3633 & 6.9136 \\
NSST & 1.0576 & 4.6326 & 6.5254 \\
\hline
\end{tabular}




\section{Abbreviations}

Cll: Contrast improvement index; ENL: Equivalent number of looks; EPI: Edge preservation index; MAT: Multi-scale analysis theories; NSCT: Non-subsampled contourlet transform; NSST: Non-subsampled shearlet transform

\section{Acknowledgements}

The authors would like to thank Tianjin Key Laboratory of Wireless Mobile Communications and Power Transmission for the support.

\section{Funding}

The funding was given by Tianjin Edge Technology and Applied Basic Research Project (14JCYBJC15800), Tianjin Normal University Doctoral Foundation (52XB1603), Tianjin Normal University Application Development Foundation (52XK1601) in China.

\section{Availability of data and materials}

The datasets supporting the conclusions of this article are included within the article (and its additional file(s)).

\section{Authors' contributions}

YT and JC conceived and designed the study. Both authors read and approved the final manuscript.

\section{Competing interests}

The authors declare that they have no competing interests.

\section{Consent for publication}

Not applicable

\section{Ethics approval and consent to participate}

Not applicable

\section{Author details}

${ }^{1}$ Tianjin Key Laboratory of Wireless Mobile Communications and Power Transmission, Tianjin Normal University, Tianjin 300387, China. ${ }^{2}$ College of Electronic and Communication Engineering, Tianjin Normal University, Tianjin 300387, China.

Received: 9 January 2017 Accepted: 27 February 2017

Published online: 09 March 2017

\section{References}

1. Q Liang, Situation understanding based on heterogeneous sensor networks and human-inspired favor weak fuzzy logic system. IEEE Syst. J. 5(2), 156-163 (2011)

2. Q Liang, X Cheng, S Huang, D Chen, Opportunistic sensing in wireless sensor networks: theory and applications. IEEE Trans. Comput. 63(8), 2002-2010 (2014)

3. T Zeng, T Zhang, W Tian, C Hu, Space-Surface Bistatic SAR image enhancement based on repeat-pass coherent fusion with Beidou-2/Compass-2 as illuminators. IEEE Geosci. Remote Sens. Lett. 13(12), 1832-1836 (2016)

4. JY Chiang, YC Chen, Underwater image enhancement by wavelength compensation and Dehazing. IEEE Trans. Image Process. 21(4), 1756-69 (2012)

5. M Liu, X Sun, H Deng, C Ye, X Zhou, Image enhancement based on intuitionistic fuzzy sets theory. IET Image Proc. 10(10), 1-9 (2016)

6. R Xiong, H Liu, X Zhang, J Zhang, S Ma, F Wu, W Gao, Image denoising via bandwise adaptive modeling and regularization exploiting nonlocal similarity. IEEE Trans. Image Process. 25(12), 5793-5805 (2016)

7. W Dong, X Wu, G Shi, Sparsity fine tuning in wavelet domain with application to compressive image reconstruction. IEEE Trans. Image Process. 23(12), 5249-5262 (2014)

8. V Bhateja, H Patel, A Krishn, A Sahu, Multimodal medical image sensor fusion framework using cascade of wavelet and Contourlet transform domains. IEEE Sensors J. 15(12), 6783-6790 (2015)

9. S Yang, W Min, L Zhao, Z Wang, Image noise reduction via geometric multiscale ridgelet support vector transform and dictionary learning. IEEE Trans. Image Process. 22(11), 4161-4169 (2013)

10. WQ Lim, The discrete shearlet transform: a new directional transform and compactly supported shearlet frames. IEEE Trans. Image Process. 19(5), 1166-80 (2010)

11. GR Easley, D Labate, WQ Lim, Sparse directional image representations using the discrete shearlet transform. Appl. Comput. Harmon. Anal. 25(1), 25-46 (2008)
12. $G$ Gao, L Xu, D Feng, Multi-focus image fusion based on non-subsampled shearlet transform. IET Image Process. 7(6), 633-639 (2013)

13. HR Shahdoosti, O Khayat, Image denoising using sparse representation classification and non-subsampled shearlet transform. SIViP 10(6), 1-7 (2016)

14. AF Laine, S Schuler, J Fan, W Huda, Mammographic feature enhancement by multi scale analysis. IEEE Trans. Med. Imaging 13(14), 725-740 (1994)

15. G Wang, L Xiao, AZ He, Algorithm research of adaptive fuzzy image enhancement in ridgelet transform domain. Acta Opt. Sin. 27(7), 1183-1190 (2007)

16. Y Tong, M Zhao, Z Wei, L Liu, Synthetic Aperture Radar image nonlinear enhancement algorithm based on NSCT transform. Physical Communication 13, 239-243 (2014)

17. Y Cheng, D Xue, X Han, An images enhancement algorithm combined with wavelet and curvelet. J Eng Graphics 30(3), 100-104 (2009)

18. SK Narayanan, RSD Wahidabanu, A View on Despeckling in Ultrasound Imaging. International Journal of Signal Processing, Image Processing and Pattern Recognition 2(3), 85-98 (2009)

19. SN Anfinsen, AP Doulgeris, T Eltoft, Estimation of the equivalent number of looks in polarimetric synthetic aperture radar imagery. IEEE Trans. Geosci. Remote Sens. 47(11), 3795-3809 (2009)

\section{Submit your manuscript to a SpringerOpen ${ }^{\circ}$ journal and benefit from:}

- Convenient online submission

- Rigorous peer review

- Immediate publication on acceptance

- Open access: articles freely available online

- High visibility within the field

- Retaining the copyright to your article

Submit your next manuscript at $>$ springeropen.com 